\title{
New molecular tools for efficient screening of cervical cancer
}

\author{
Magnus von Knebel Doeberitz \\ Division of Molecular Diagnostics \& Therapy, \\ Department of Surgery, University of Heidelberg, Im \\ Neuenheimer 110, D-69120 Heidelberg, Germany \\ Tel.: +4962215628 76; Fax: +496221565981; \\ E-mail: knebel@med.uni-heidelberg.de and \\ mvkd@aol.com
}

Cytological screening using the Pap-smear led to a remarkable reduction of the mortality of cervical cancer. However, due to subjective test criteria it is hampered by poor interand intra-observer agreement. More reproducible assays are expected to improve the current screening and avoid unnecessary medical intervention and psychological distress for the affected women. Cervical cancer arises as consequence of persistent high risk papillomavirus (HR-HPV) infections. Expression of two viral oncogenes, E6 and E7, in epithelial stem cells is required to initiate and maintain cervical carcinogenesis and results in significant overexpression of the cellular p16INK4a protein. Since this protein is not expressed in normal cervical squamous epithelia, screening for $\mathrm{p} 16 \mathrm{INK} 4 \mathrm{a}$ over-expressing cells allows to specifically identify dysplastic lesions, and significantly reduces the inter-observer disagreement of the conventional cytological or histological tests. Progression of preneoplastic lesions to invasive cancers is associated with extensive recombination of viral and cellular genomes which can be monitored by detection of papillomavirus oncogene transcripts (APOT assay) derived from integrated viral genome copies. Detection of integrated type oncogene transcripts points to far advanced dysplasia or invasive cancers and thus represents a progression marker for cervical lesions. These new assays discussed here will help to improve current limitations in cervical cancer screening, diagnosis, and therapy control.

\section{Introduction}

\subsection{Cytological screening for cervical cancer}

Cervical cancer is still the second most frequent neoplasm in women world wide [1]. There are about 450000 new cases each year which cause death of about 250000 women annually, mostly in developing countries [2]. Cytological staining of simple cervical smears was developed by George Papanicolaou (Pap-smear) in the 1930's [3] and its introduction in population wide screening programs in most industrialized societies led to a remarkable reduction of the incidence and mortality of cervical cancer [4]. In patients with suspicious smears, biopsies are taken from dysplastic epithelial regions to establish a histopathological diagnosis which then guides further treatment options [5]. In fact, up to now, there is no other comparable diagnostic or therapeutic intervention which reduced the mortality of any neoplastic disease to a comparable extent. Despite these convincing achievements, this screening approach is hampered by highly subjective morphological parameters and low inter- and intra-observer agreement, particularly in the interpretation of low-grade lesions in both cytology and cervical biopsies [6-8]. This leads to many repeated tests, overand undertreatment, and significant emotional distress for the affected patients, as well as high unnecessary costs for the health care providers $[9,10]$. The reduction of cervical cancer mortality is thus achieved by accepting many repeated tests and eventually unnecessary treatment in many women with suspicious equivocal screening tests. A more objective screening test based, for example, on a specific biomarker which was only expressed in dysplastic cervical epithelia but not in normal non-transformed cervical cells, would allow unambiguous identification of dysplastic cells. Consequently, this would result in reduction of costs spend for unnecessary surgical intervention, and in psychological distress from screening and unnecessary treatments.

\section{High risk human papillomaviruses as causative agents of cervical cancer}

Many lines of evidence clearly demonstrated that persistent infections with high risk group human papillomaviruses (HR-HPV) are essential but not exclusive prerequisites for the initiation of cervical carcinogen- 


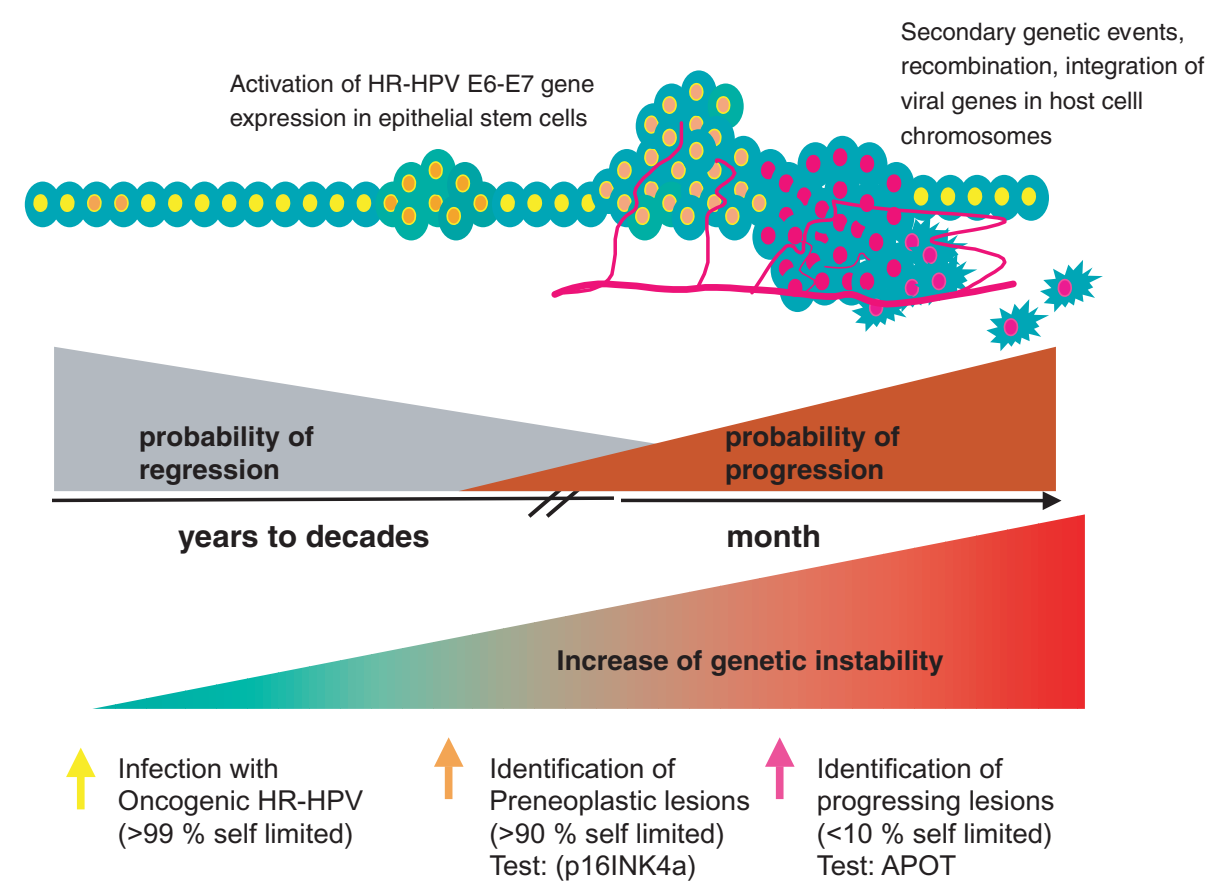

Fig. 1. Schematic representation of cervical carcinogenesis. HR-HPV infections are usually self-limited and resolve spontaneously. Only few persist and induce a dysplastic lesion. With progressing genetic alterations of the HR-HPV infected cells, increasing levels of the HR-HPV oncogenes E6 and E7 are expressed, which results in increasing expression of the p16INK4a protein (see also Fig. 2). Additional genomic alterations finally result in integration of the viral genome and high level expression of the E6-E7 oncogenes encoded by integrated viral genome copies. This can be monitored with the APOT assay (see Fig. 3).

esis [11]. In fact, depending on the age group more than 20 percent of the female population are infected with human papillomaviruses in the genital tract [12] (Fig. 2), only very few of whom will eventually develop persistent infections and subsequently cervical dysplasia. There is good evidence that most asymptomatic HPV-infections as well as early dysplastic lesions (CIN 1) regress spontaneously [13] and even among the advanced CIN 2/CIN 3 lesions it is estimated that most persist or regress and that only very few progress to invasive cancer [14]. Since almost all cervical cancers however, harbour HR-HPV genomes [15], this suggests that HR-HPV infections constitute an initial key event in cervical carcinogenesis, but additional secondary genetic events are required for the progression to dysplasia or invasive cancers [16] (Fig. 1).

\section{Expression of the HR-HPV oncogenes E6-E7 is required to induce and maintain cervical dysplasia and cancer}

Progression of cervical dysplasia to advanced stages or invasive cancers is linked to increasing genetic in- stability. This is reflected by many chromosomal aberrations [16] and requires the expression of two papillomavirus oncogenes, E6 and E7, which in "normal" HR-HPV infected lesions is highly suppressed by intracellular control mechanisms [17]. Loss of this intracellular control of viral gene expression permits the expression of viral oncogenes. Due to their inhibition of various cellular cell cycle regulators this results in a significant reduction of control functions of the G1/Sphase transition in the cell cycle, presumably allowing cells to enter the S-phase without prior proof-reading of their genomes and thus accumulation of mutations in the respective daughter cells [18-20]. Enhanced expression of the viral oncogenes is directly linked to the cell kinetics of HR-HPV transformed cells and reduction of the activity of the viral oncogenes, results in reduced cell growth and loss of the neoplastic properties of cervical cancer cells [21-23].

\section{P16INK4a as biomarker to highlight cells which express the viral oncogenes E6 and E7}

Expression of the viral oncogenes in epithelial stem cells of the uterine cervix is thus the critical gate keeper 


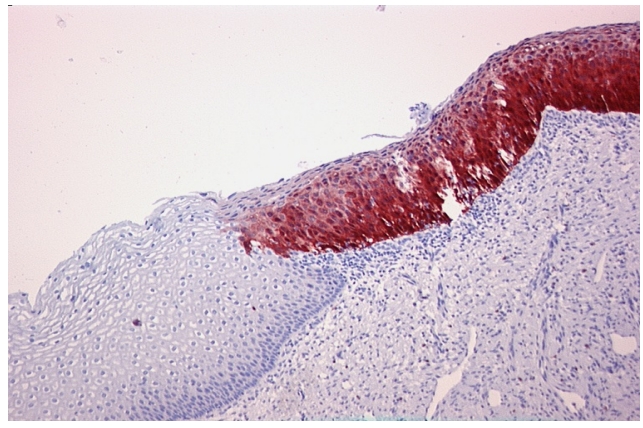

A

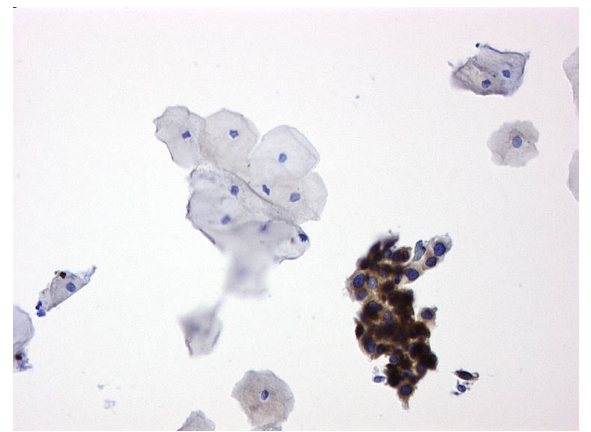

Fig. 2. a) Immunohistochemical staining of a high grade cervical intraepithelial neoplasia with the anti-p16INK4a monoclonal antibody E6H4 (MTM Laboratories AG, Heidelberg, Germany). b) Immunocytochemistry of cytospin preparations of cervical cells collected with a cytobrush using the anti-p16INK4a monoclonal antibody E6H4 (MTM Laboratories AG, Heidelberg, Germany).

event in cervical carcinogenesis. It was therefore reasonable to assume that monitoring for the expression level of the viral oncogenes in epithelial stems might be a suitable biomarker for dysplastic cells. However, due to many technical obstacles it was not possible to established robust and simple clinical applicable assays. We therefore set out to identify appropriate cellular surrogate markers, which reflect the expression of the viral oncogenes in infected epithelial stem cells. Surprisingly, among many markers tested, high levels of the p16INK4a gene product could be identified in almost all dysplastic cervical lesions by a new set of highly specific monoclonal antibodies [24,25], whereas it was not expressed in squamous epithelial and endocervical cells of the lower genital tract and only few restricted endometrial epithelia. This highly restricted expression pattern in normal epithelia and the strong and increasing overexpression in dysplastic lesions of the cervix suggested that using p16INK4a immunohistochemistry indeed allows much more precisely to identify dysplastic cells in biopsy samples or even cytological smears (Fig. 2).

In an international assessment study we aimed to define the contribution of p16-based immunohistochemistry to decrease the interobserver variation in the interpretation of histological slides obtained from cervical biopsies or cones (Klaes et al., submitted). This study revealed a significant discrepancy in the interpretation of $H \& E$ stained slides of cervical biopsies. In particular, lesions classified as low or borderline dysplasia showed the greatest discrepancies in the interpretation among a panel of five expert gynecopathologists. Corresponding slides were stained with the p16INK4a specific monoclonal antibody. The interpretation of these slides was much more uniform and revealed much less interobserver disagreement. Thus, using p16INK4a based immunohistochemistry significantly reduces the discrepant interpretation of histological slides of dysplastic cervical lesions.

Using p16INK4a as primary screening test in cytology and as specific stain in histology to identify dysplastic cells will clearly improve the quality of the conventional diagnostic tests, however, it does not provide information on the potential progression risk of the respective cervical lesions. Thus, additional markers, which allow to assess the potential progression risk of cervical lesions would be clinically helpful to come to definitive therapeutic recommendations.

\section{Amplification of papillomavirus oncogene transcripts (APOT) derived from integrated viral genomes as progression marker for advanced dysplasia and invasive cancers}

Many dysplastic cervical lesions persist over month and years and eventually spontaneously regress, and only few progress over a short period of time to invasive cancers. There are no morphological parameters which would allow to predict this strikingly different biological behaviour. From the clinical point of view, it would be very helpful, if precise parameters would allow to differentiate lesions which rapidly will progress from those that still retain the capacity to regress. Enhanced genetic instability reflected by more extensive recombination of genomic fragments in a cancer cell or its precursors is believed to provide an ample reservoir for genetic flexibility. In HR-HPV associated lesions recombination of viral and cellular genomes is frequently observed in advanced lesions and almost regularly in 
A
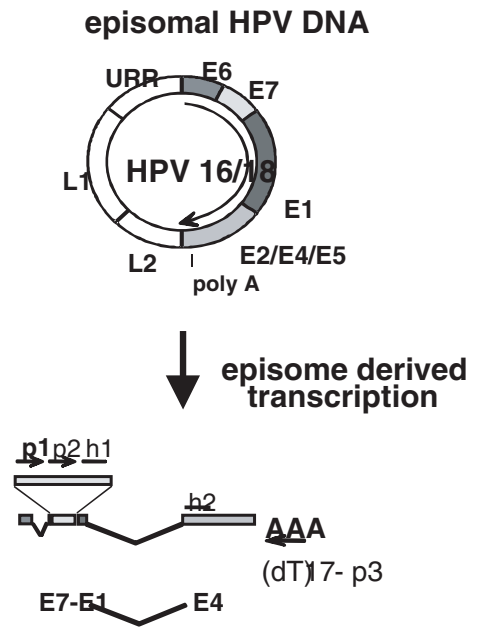

integrated HPV DNA

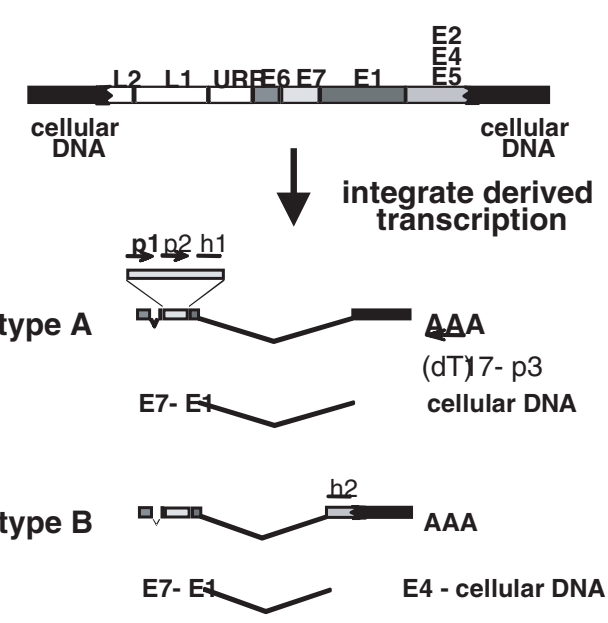

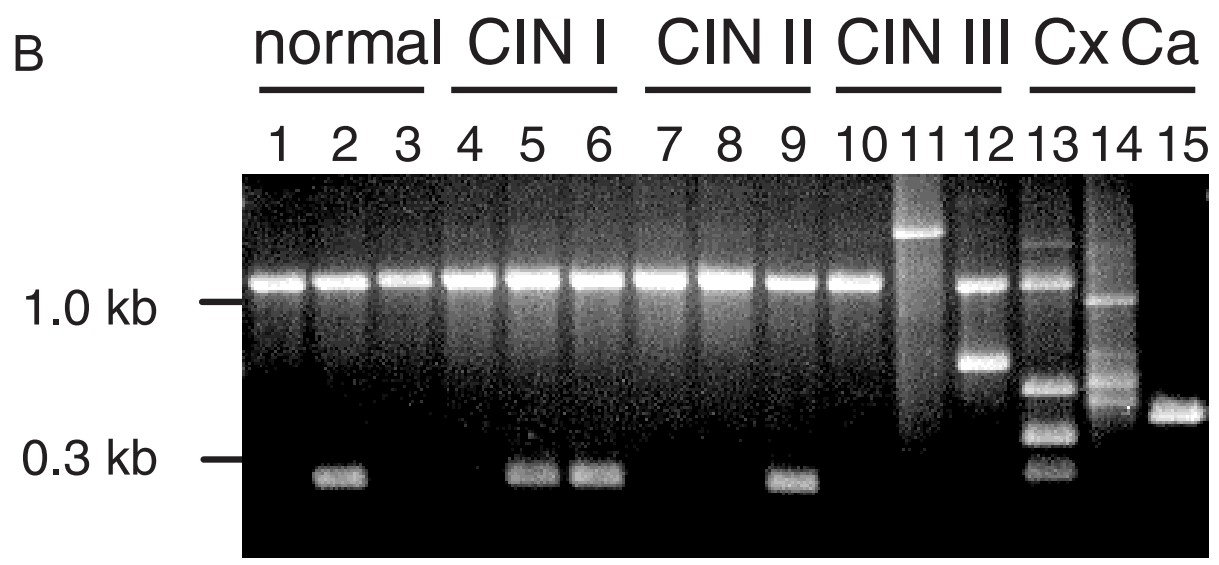

Fig. 3. a) Structure of HPV genomes, either in their episomal (left) or integrated form (right), and transcripts derived thereof. E6-E7 oncogene transcripts are amplified by starting with a RT-PCR reaction using oligo dT linked primers $\left.((\mathrm{dT}))_{7}-\mathrm{p} 3\right)$. The respective cDNA are subsequently amplified using a specific primer linked to the oligo dT tail ((dT) $\left.{ }_{17}-\mathrm{p} 3\right)$ and E7 specific primers (p1, p2, p3). The protocol is described in detail in [30]. Integrate derived transcripts differ either in lacking (type A) or containing E4 sequences (type B). Episomal or integrated transcripts can be differentiated by size differentiation (Fig. 3(b)). b) Amplification of papillomavirus oncogene transcripts (APOT) from HPV16-positive clinical samples of normal cervical epithelia, preneoplastic lesions (CIN I, II, III), and primary cervical carcinomas (CxCa). RT-PCR products were separated on agarose gels. Note the consistent size of the amplimere in lanes 1-10 (episome derived transcripts) and different sizes of the amplimeres in lanes 11-15 (integrate derived transcripts).

invasive cancers, whereas in early preneoplasia HRHPV genomes were usually found to persist as episomal genomes [26]. Integration of viral genomes moreover was shown to result in enhanced expression of viral oncogenes due to deregulated cellular control of the viral oncogenes [27] as well as to enhanced stability and consequently enhanced expression levels of the viral oncogenes [28,29]. Taken together, these findings suggested that lesions which display integrated viral genomes are at significantly higher risk for neoplas- tic progression compared to those in which the viral genome persists as extra-chromosomal episome.

A new RT-PCR based technique allows to identify HR-HPV oncogene transcripts derived from integrated viral genomes and thus to differentiate these from transcripts derived from episomal viral genomes (amplification of papillomavirus oncogene transcripts, APOTassay) (Figs 3(a) and (b)) [30]. Using this assay, "integrated type" HR-HPV oncogene transcripts were detected in a prospective study in $5 \%$ of patients with CIN 2 lesions, $16 \%$ of patients with CIN 3 lesions and 
$85 \%$ of patients with invasive cervical cancers. These data strongly suggest, that detection of integrated HRHPV oncogene transcripts point to advanced preneoplastic lesions or already invasive cervical cancers [30]. Patients with positive APOT assays therefore require careful clinical work-up to locate and treat adequately the underlying lesions. Moreover, from various patients, in whom positive APOT test results suggested integrated viral genomes, repeated smears or biopsies were obtained during follow-up visits after therapy. In these patients clinical relapses could be predicted by persisting positive APOT tests. Interestingly, in most of these patients, identical APOT fragments were amplified. This indicates, that the integration site of the viral genome was identical in both lesions and suggests a common clonal origin of both lesions. Thus, it is reasonable to assume that in these patients rather persisting dysplastic cells caused the clinical relapse instead of persisting HPV infections which caused repeated transformation of new cell clones. The APOT assay is therefore a valuable tool to monitor therapy efficacy after surgical treatment of HR-HPV induced dysplasia in the female lower genital tract and to precisely predict clinical relapses after initial therapy.

\section{Conclusions}

The experimental dissection of the molecular pathways contributing to cervical carcinogenesis allowed to predict potential new biomarkers which would improve the cytological and histological diagnosis of cervical lesions. In particular overexpression of p16INK4a is regularly observed in cervical lesions induced by HR-HPV infection, but absent in normal cervical epithelia or lesions induced by low risk HPV types. Implementing staining with p16INK4a specific antibodies in cytology and histology would clearly improve the inter- and intraobserver agreement and leads to less repeated tests and avoids unnecessary further diagnostic or therapeutic interventions. It therefore will presumably also lead to significant cost reduction in cervical cancer screening. The detection of integrated HR-HPV genomes using the APOT assay points to advanced preneoplastic lesions or invasive cervical cancers. It might prove as useful parameter to better assess the progression risk of cervical lesions and might help in the surveillance of patients after therapy to predict relapses of the disease much earlier than it is possible with the conventional techniques.

\section{Acknowledgment}

This work was supported by grants of the Deutsche Krebshilfe (M 13/95/Kn1, 70-2449-Kn3).

\section{References}

[1] D.M. Parkin, P. Pisani and J. Ferlay, Estimates of the worldwide incidence of 25 major cancers in 1990, Int. J. Cancer $\mathbf{8 0}$ (1999), 827-841.

[2] P. Pisani, D.M. Parkin, F. Bray and J. Ferlay, Estimates of the worldwide mortality from 25 cancers in 1990 [published erratum appears in Int J Cancer 1999 Dec 10;83(6):870-873], Int. J. Cancer 83 (1999), 18-29.

[3] G.N. Papanicolaou and H.F. Traut, The diagnostic value of vaginal smears in carcinoma of the uterus. 1941 [classical article], Arch. Pathol. Lab Med. 121 (1997), 211-224.

[4] N. Segnan, Cervical cancer screening. Human benefits and human costs in the evaluation of screening programmes, Eur. J. Cancer 30A (1994), 873-875.

[5] R.J. Kurman, D.E. Henson, A.L. Herbst, K.L. Noller and M.H. Schiffman, Interim guidelines for management of abnormal cervical cytology. The 1992 National Cancer Institute Workshop, JAMA 271 (1994), 1866-1869.

[6] W.G. McCluggage, M.Y. Walsh, C.M. Thornton, P.W. Hamilton, A. Date and L.M. Caughley et al., Inter- and intra-observer variation in the histopathological reporting of cervical squamous intraepithelial lesions using a modified Bethesda grading system, Br. J. Obstet. Gynaecol. 105 (1998), 206-210.

[7] S.M. Ismail, A.B. Colclough, J.S. Dinnen, D. Eakins, D.M. Evans and E. Gradwell et al., Reporting cervical intraepithelial neoplasia (CIN): intra- and interpathologist variation and factors associated with disagreement, Histopathology 16 (1990), 371-376.

[8] P.D. Sasieni, J. Cuzick and E. Lynch-Farmery, Estimating the efficacy of screening by auditing smear histories of women with and without cervical cancer. The National Co-ordinating Network for Cervical Screening Working Group, Br. J. Cancer 73 (1996), 1001-1005.

[9] M.H. Jones, A. Singer and D. Jenkins, The mildly abnormal cervical smear: patient anxiety and choice of management, $J$. R. Soc. Med. 89 (1996), 257-260.

[10] C. Sherlaw-Johnson, S. Gallivan, D. Jenkins and M.H. Jones, Cytological screening and management of abnormalities in prevention of cervical cancer: an overview with stochastic modelling, J. Clin. Pathol. 47 (1994), 430-435.

[11] H. zur Hausen, Papillomaviruses in human cancers, Proc. Assoc. Am. Physicians 111 (1999), 581-587.

[12] T.Y. Chu, C.Y. Shen, H.S. Lee and H.S. Liu, Monoclonality and surface lesion-specific microsatellite alterations in premalignant and malignant neoplasia of uterine cervix: a local field effect of genomic instability and clonal evolution, Genes Chromosomes. Cancer 24 (1999), 127-134.

[13] G.Y. Ho, R. Bierman, L. Beardsley, C.J. Chang and R.D. Burk, Natural history of cervicovaginal papillomavirus infection in young women, N. Engl. J. Med. 338 (1998), 423-428.

[14] A.G. Ostor, Natural history of cervical intraepithelial neoplasia: a critical review, Int. J. Gynecol. Pathol. 12 (1993), 186-192.

[15] J.M. Walboomers, M.V. Jacobs, M.M. Manos, F.X. Bosch, J.A. Kummer and K.V. Shah et al., Human papillomavirus is 
a necessary cause of invasive cervical cancer worldwide, $J$. Pathol. 189 (1999), 12-19.

[16] P.A. Lazo, The molecular genetics of cervical carcinoma, $\mathrm{Br}$. J. Cancer 80 (1999), 2008-2018.

[17] H.H. Zur, Papillomaviruses causing cancer: evasion from host-cell control in early events in carcinogenesis, J. Natl. Cancer Inst. 92 (2000), 690-698.

[18] C. Sherlaw-Johnson, S. Gallivan and D. Jenkins, Withdrawing low risk women from cervical screening programmes: mathematical modelling study [see comments], BMJ 318 (1999), 356-360.

[19] C. Sherlaw-Johnson, S. Gallivan and D. Jenkins, Evaluating cervical cancer screening programmes for developing countries, Int. J. Cancer 72 (1997), 210-216.

[20] M.P. O'Connell, D.M. Jenkins, A.W. Curtain, P.A. Hughes and J. Doyle, Benign cervical leiomyoma leading to disseminated fatal malignancy, Gynecol. Oncol. 62 (1996), 119-122.

[21] M. von Knebel Doeberitz, T. Oltersdorf, E. Schwarz and L. Gissmann, Correlation of modified human papilloma virus early gene expression with altered growth properties in C4-1 cervical carcinoma cells, Cancer Res. 48 (1988), 3780-3786.

[22] M. von Knebel Doeberitz, C. Rittmuller, H. zur Hausen and M. Durst, Inhibition of tumorigenicity of cervical cancer cells in nude mice by HPV E6-E7 anti-sense RNA [letter], Int. J. Cancer 51 (1992), 831-834.

[23] M. von Knebel Doeberitz, C. Rittmuller, F. Aengeneyndt, P. Jansen-Durr and D. Spitkovsky, Reversible repression of papillomavirus oncogene expression in cervical carcinoma cells: consequences for the phenotype and E6-p53 and E7-pRB interactions, J. Virol. 68 (1994), 2811-2821.
[24] T. Sano, T. Oyama, K. Kashiwabara, T. Fukuda and T. Nakajima, Expression status of p16 protein is associated with human papillomavirus oncogenic potential in cervical and genital lesions, Am. J. Pathol. 153 (1998), 1741-1748.

[25] R. Klaes, T. Friedrich, D. Spitkovsky, R. Ridder, W. Rudy and K.U. Petry et al., Overexpression of p16 INK4a as specific marker for dysplastic and neoplastic epithelial cells of the cervix Uteri, Int. J. Cancer (2001), accepted for publication.

[26] A.P. Cullen, R. Reid, M. Campion and A.T. Lorincz, Analysis of the physical state of different human papillomavirus DNAs in intraepithelial and invasive cervical neoplasm, J. Virol. 65 (1991), 606-612.

[27] M. von Knebel Doeberitz, T. Bauknecht, D. Bartsch and H.H. zur, Influence of chromosomal integration on glucocorticoidregulated transcription of growth-stimulating papillomavirus genes E6 and E7 in cervical carcinoma cells, Proc. Natl. Acad. Sci. USA 88 (1991), 1411-1415.

[28] S. Jeon, B.L. Allen-Hoffmann and P.F. Lambert, Integration of human papillomavirus type 16 into the human genome correlates with a selective growth advantage of cells, J. Virol. 69 (1995), 2989-2997.

[29] S. Jeon and P.F. Lambert, Integration of human papillomavirus type 16 DNA into the human genome leads to increased stability of E6 and E7 mRNAs: implications for cervical carcinogenesis, Proc. Natl. Acad. Sci. USA 92 (1995), 1654-1658.

[30] R. Klaes, S.M. Woerner, R. Ridder, N. Wentzensen, M. Duerst and A. Schneider et al., Detection of high-risk cervical intraepithelial neoplasia and cervical cancer by amplification of transcripts derived from integrated papillomavirus oncogenes, Cancer Res. 59 (1999), 6132-6136. 


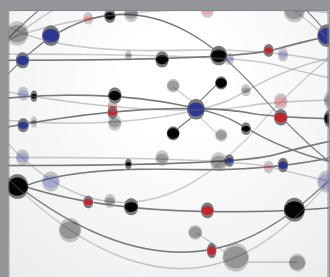

The Scientific World Journal
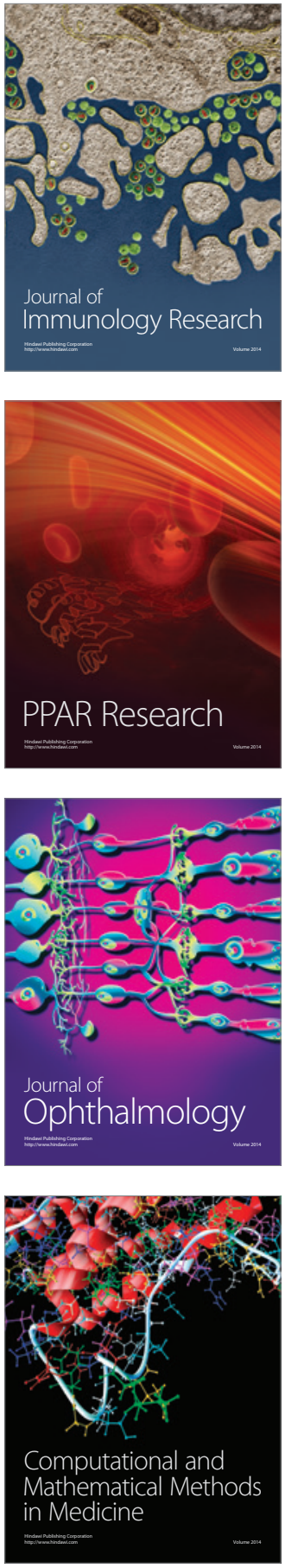

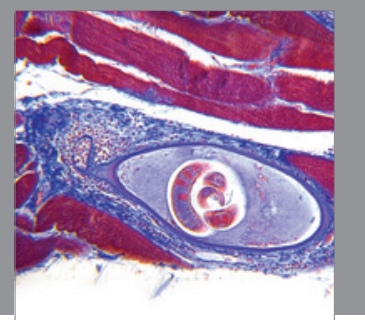

Gastroenterology

Research and Practice
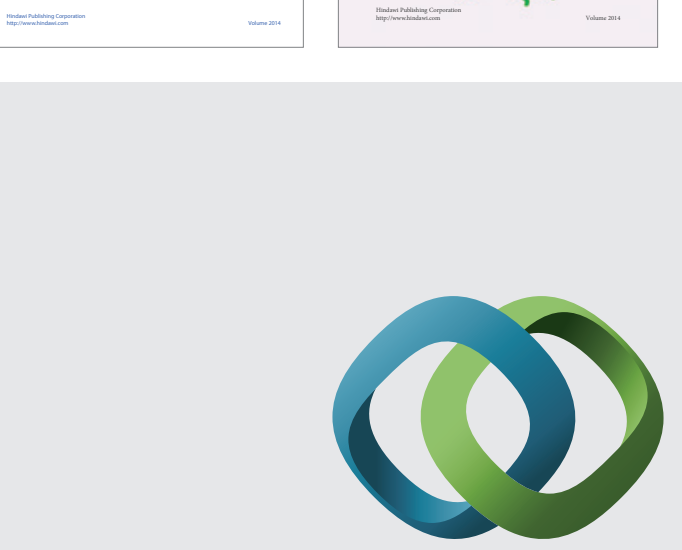

\section{Hindawi}

Submit your manuscripts at

http://www.hindawi.com
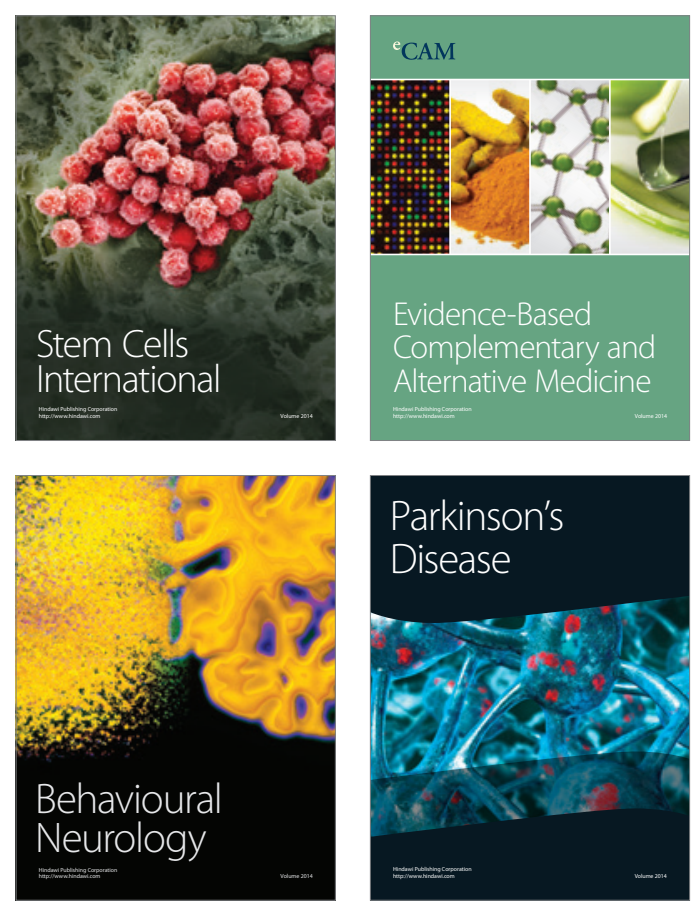

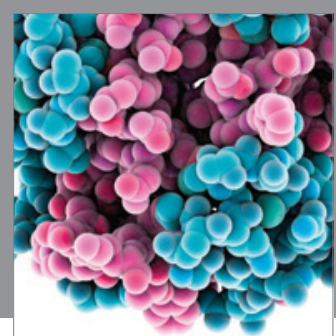

Journal of
Diabetes Research

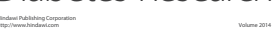

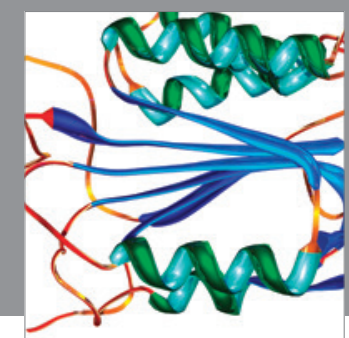

Disease Markers
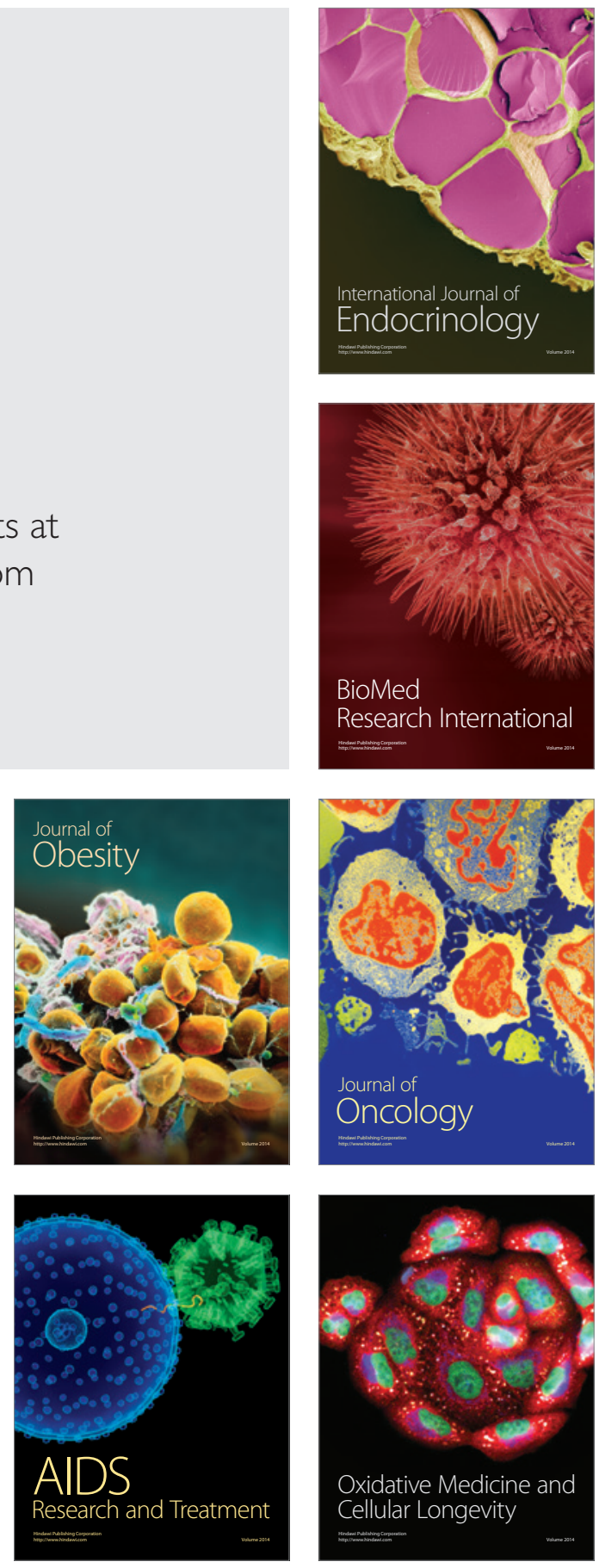